\title{
Experimental Control and Characterization of Autophagy in Drosophila
}

\author{
Gabor Juhasz and Thomas P. Neufeld
}

\begin{abstract}
Summary
Insects such as the fruit fly Drosophila melanogaster, which fundamentally reorganize their body plan during metamorphosis, make extensive use of autophagy for their normal development and physiology. In the fruit fly, the hepatic/adipose organ known as the fat body accumulates nutrient stores during the larval feeding stage. Upon entering metamorphosis, as well as in response to starvation, these nutrients are mobilized through a massive induction of autophagy, providing support to other tissues and organs during periods of nutrient deprivation. High levels of autophagy are also observed in larval tissues destined for elimination, such as the salivary glands and larval gut. Drosophila is emerging as an important system for studying the functions and regulation of autophagy in an in vivo setting. In this chapter we describe reagents and methods for monitoring autophagy in Drosophila, focusing on the larval fat body. We also describe methods for experimentally activating and inhibiting autophagy in this system and discuss the potential for genetic analysis in Drosophila to identify novel genes involved in autophagy.
\end{abstract}

Key Words: Atg8; autophagy; Drosophila; GFP; TEM; LysoTracker Red; somatic clones.

\section{Introduction}

Autophagic structures in insect tissues were first described nearly 40 years ago in larval cells preparing for metamorphosis (reviewed in ref. 1). More recent analyses in Drosophila have focused on the interactions of autophagic and apoptotic processes (2-9), the regulation of autophagy by nutrient and hormonal signaling pathways (9-12), and its role in neurodegeneration (13). As Drosophila has emerged as a genetically amenable system to analyze 
autophagy in vivo, a description of currently used methods for manipulating and monitoring autophagy in this system may be of use. Here we describe a number of approaches applicable to the study of autophagy in Drosophila.

\section{Materials}

\subsection{Drosophila Stocks}

For clonal expression of transgenes, we cross UAS lines of interest carrying $\mathrm{X}$-linked hsp70-FLP to a Act>CD2>GAL4 UAS-GFP strain. For loss of function mosaic studies, mutations of interest are recombined with FRT inserts at the base of the proper chromosome arm, then crossed with fb-GAL4 FRT UAS-GFP lines. Alternatively, clones can be marked using UAS-dsRed or UAS-RFP, which allows GFP-Atg8 to be used as an autophagy indicator in this system.

\subsection{Larval Cultures and Starvation}

1. Standard cornmeal-molasses-agar fly food.

2. $20 \%$ sucrose in water.

\subsection{Dissection and Staining of Fat Bodies with LysoTracker Red}

1. PBS (phosphate-buffered saline), $\mathrm{pH}=7.4$.

2. DAPI (4',6-diamidino-2-phenylindole dihydrochloride; Sigma, St. Louis, MO) dissolved in water to obtain a $1 \mathrm{~m} M(1000 \mathrm{x})$ stock solution, which can be stored in a dark vial at $4^{\circ} \mathrm{C}$.

3. LysoTracker Red DND-99 (Molecular Probes, Eugene, OR) diluted in PBS to obtain a $20 \mathrm{~m} M(200 \mathrm{x})$ stock solution, which is stable in a dark vial at $4^{\circ} \mathrm{C}$ for a few weeks.

\subsection{Fixation for Confocal Microscopy}

1. Formaldehyde (Fluka, Milwaukee, WI).

2. Vectashield (Vectorlabs, Burlingame, CA).

\subsection{Fixation and Embedding for Transmission Electron Microscopy (TEM)}

1. Paraformaldehyde, glutaraldehyde, $\mathrm{OsO}_{4}$, Polybed 812 (Polysciences, Warrington, PA). 


\section{Methods}

\subsection{Experimental Regulation of Autophagy in the Larval Fat Body}

This section discusses approaches for experimentally inducing or inhibiting autophagy in the Drosophila larval fat body, using manipulations of culture conditions and genetic expression.

\subsubsection{Nutrient Control}

The larval fat body is exquisitely sensitive to nutrient conditions, and thus autophagy is readily induced by a simple starvation regimen. Induction of autophagy can first be detected within $1-2 \mathrm{~h}$ of starvation, and by $4 \mathrm{~h}$ after food withdrawal a robust response is consistently observed.

\subsubsection{Pretreatment}

Due to the high sensitivity of the fat body to nutrient levels, preincubation of larvae in fresh media under uncrowded conditions is recommended to prevent chronic induction of autophagy, which can diminish the acute response to starvation. We generally transfer 10-20 late second-instar larvae to vials containing fresh fly food supplemented with live yeast paste, $16-24 \mathrm{~h}$ prior to starvation.

\subsubsection{InduCtion by Starvation}

Larvae can be conveniently floated out of fly food media by pouring several $\mathrm{mL}$ of a $20 \%$ sucrose solution onto the culture; the high density of this solution causes the larvae to float to the surface. Larvae are then transferred by forceps to vials or small dishes containing fresh $20 \%$ sucrose and incubated at room temperature for the duration of the starvation period, generally $4 \mathrm{~h}$. Alternatively, larvae can be transferred to vials containing a strip of filter paper saturated with PBS. Following the starvation period, larvae are dissected and immediately processed as described in section 3.2.2.

\subsubsection{InDUCTION BY RAPAMYCIN}

The autophagic response to starvation in the larval fat body appears to result largely from inhibition of the target of rapamycin (TOR) signaling pathway (14). As an alternative to starvation, direct inhibition of TOR by the drug rapamycin results in a robust autophagic response. Following pretreatment as above, larvae are transferred to fresh fly food containing rapamycin at a concentration of $1 \mu M$. Weak induction is observed by $4 \mathrm{~h}$, with a much stronger response occurring by $24 \mathrm{~h}$. 


\subsubsection{Developmental Control}

During the final $24 \mathrm{~h}$ of the larval period, rising titers of the steroid hormone ecdysone result in a developmentally programmed induction of autophagy in the larval fat body $(\mathbf{1 2 , 1 5})$. This developmentally regulated autophagy can be readily observed by comparing autophagy markers in early vs. late third-instar larvae under fed conditions. Premature induction can be achieved by exposure of dissected fat body to ecdysone in culture. Such methods have been described previously (15) and are not detailed here.

\subsubsection{Genetic Control}

A major advantage of Drosophila is the ease with which genetic function can be manipulated in this system. Gene expression can be readily induced or inhibited in vivo, either singly or in multiple combinations, and at precise times and locations during development. An important component of this control is the ability to alter gene expression using a mosaic approach, in which marked clones of cells differ in their genetic makeup from their wild-type neighbors (Fig. 1A,B). The principal advantage of this approach is that experimental and control cells are subject to the same environmental and developmental conditions, and thus subtle phenotypic differences can be more readily detected than in animal-to-animal comparisons. This technique is especially applicable to studies of autophagy, which vary with developmental and culture conditions.

The method of choice for both loss and gain of function mosaic experiments in Drosophila is the Flippase (FLP)-Flippase Recombinase Target (FRT) system of mitotic recombination. In this system, FLP-induced exchange between heterozygous sister chromatids causes loss of heterozygosity for a given chromosomal arm. Alternatively, FLP-induced expression of the GAL4 transcription factor can be used to clonally activate UAS-controlled transgenes. As these methods have been extensively reviewed elsewhere $(\mathbf{1 6}, 17)$, we will focus our comments on their application in the larval fat body.

\subsubsection{LosS OF FunCtion Clones}

The induction of FLP-mediated, green fluorescent protein (GFP)-marked loss of function clones in the fat body requires two modifications of the general protocols developed for imaginal tissues. First, because this procedure works only in mitotically active cells, clones must be induced during the first $8 \mathrm{~h}$ of embryonic development, after which time the fat body enters an endoreduplicative (nonmitotic) cell cycle. We typically perform 6- to 8-h embryo collections, followed immediately by a 1 - to 2 -hour heat shock at $37^{\circ} \mathrm{C}$ to activate expression of the hsp70-driven FLP gene. This typically gives rise to several 


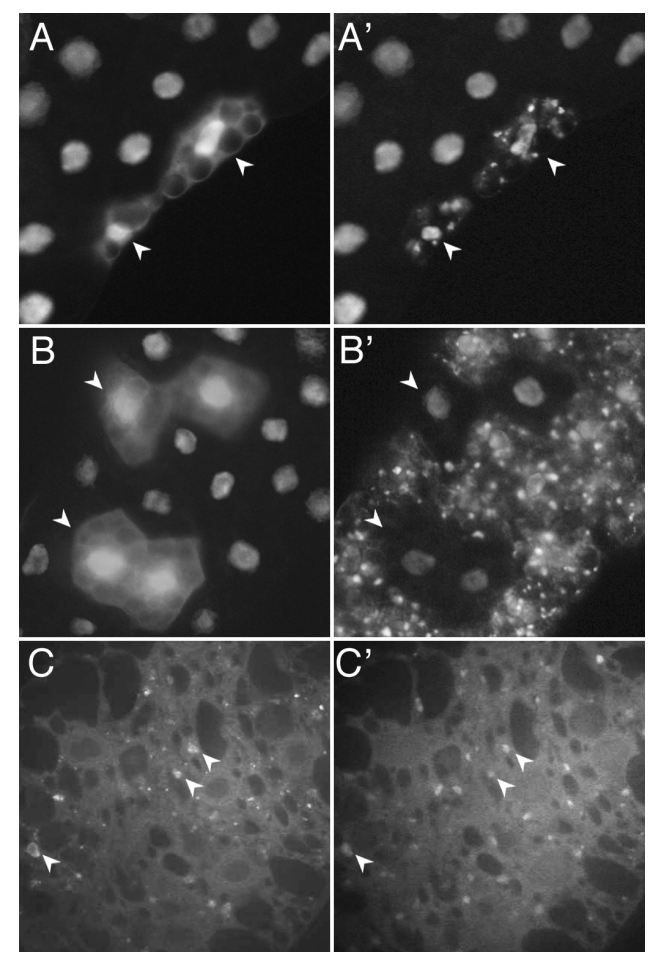

Fig. 1. Autophagy in the larval fat body. Examples of Lysotracker Red-stained samples are shown. (A, $\left.\mathbf{A}^{\prime}\right)$ In fed animals, clonal overexpression of Tsc1 and Tsc2, negative regulators of the TOR signaling pathway, causes a punctate pattern of LysoTracker Red staining $\left(\mathbf{A}^{\prime}\right)$ exclusively within the transgene-expressing cells, which are marked by cytoplasmic GFP expression in (A) and indicated by arrowheads. Nuclei are stained with DAPI. (B, $\left.\mathbf{B}^{\prime}\right)$ In response to a 4-h starvation treatment, wild-type control cells display strong punctate LysoTracker Red staining $\left(\mathbf{B}^{\prime}\right)$. In contrast, LysoTracker Red staining is blocked in cells expressing high levels of Rheb, an upstream activator of TOR signaling. Cells overexpressing Rheb are marked by cytoplasmic GFP expression in (B) and indicated by arrowheads. Nuclei are stained with DAPI. (C, $\mathbf{C}^{\prime}$ ) Fat body dissected from 4-h starved larvae expressing GFP-Atg8 (C) and stained with LysoTracker Red $\left(\mathbf{C}^{\prime}\right)$. Large punctate structures positive for both GFP-Atg8 and LysoTracker Red represent autolysosomes (indicated by arrowheads), whereas smaller structures marked solely by GFP-Atg8 represent autophagosomes.

one- to two-cell clones per fat body lobe. Second, we find that the commonly used GFP marker lines driven by the ubiquitin promoter display nonuniform expression levels in the fat body, which can confound efforts to identify clones by GFP expression. As a remedy, we use Upstream Activating Sequence (UAS)- 
driven GFP under the control of strong fat body GAL4 lines such as fb- or Cg-GAL4 $(9,18)$. These transgenes are recombined onto chromosomes bearing centromere-proximal FRT sites prior to use. A final note is that, unlike in imaginal tissues, fat body cells mix extensively during development, which often results in considerable separation of clones from their sister twinspots.

\subsubsection{Clonal Expression of Transgenes}

A unique feature of the fat body makes it especially amenable to FLPmediated clonal induction of transgene expression. Britton et al. (19) described heat shock-independent spontaneous induction of "flip-out" clones in the fat body and midguts of hsp70-FLP Act>CD2>GAL4-bearing larvae. Presumably these tissues are more sensitive than others to low levels of leaky expression of FLP early in their development. Induction of FLP later in development is not practical in the fat body, as it tends to result in ubiquitous transgene expression throughout the fat body, likely due to the polyploid nature of this tissue. Figure 1 displays examples of spontaneously induced clones of GFPmarked cells overexpressing Tsc1 and Tsc2 (A), which induce autophagy by inhibiting TOR, or Rheb (B), which suppresses starvation-induced autophagy by maintaining TOR activation.

\subsubsection{Forward Genetic Approaches to IDentifying AutOphagy Regulators}

It is perhaps obvious to point out that the genetic approaches discussed above can easily be adapted for use as tools in gene discovery. The development of comprehensive collections of UAS-regulated lines allowing induction of overexpression or RNA interference will undoubtedly lead to identification of novel regulators and effectors of autophagy. In addition, newly available collections of FRT-linked lethal P insertions provide a valuable resource for loss of function mosaic studies.

\subsection{Monitoring Autophagy in the Larval Fat Body}

Here we describe three independent methods for assaying autophagy in the fat body. LysoTracker Red stains acidic organelles in fixed and living cells and is usually confined to the late endosomal and lysosomal compartments. Under fed conditions, the larval fat body displays virtually no punctate LysoTracker Red staining. Starvation causes a robust induction of staining, which is severely inhibited in autophagy mutants. Thus, LysoTracker Red can be considered a reliable indicator of starvation-induced autophagy in this tissue. Examples of LysoTracker Red staining are shown in Fig. 1. Greater specificity can be achieved by following the localization of GFP-Atg8, which becomes conjugated 
to autophagosomal membranes. Lines expressing GFP fused to both human and fly homologs of Atg8 have been described $(10,12)$ and are available. The combination of LysoTracker Red staining and GFP-Atg8 fluorescence allows distinction between autophagosomes (green signal only) and autolysosomes (both green and red signal) (Fig. 1C). Finally, we include a brief protocol for analysis by TEM.

\subsubsection{Larval Cultures and Starvation}

1. Set up fly crosses with 5-10 females and a similar number of males. Do a 24-h egg collection.

2. Forty-eight hours after finishing egg collection, transfer approximately 20 L2stage larvae to a fresh vial to reduce crowding. Add yeast paste or dry yeast to make sure all larvae have plenty of food to eat.

3. On the next day, collect larvae to examine (fed), or transfer 8-10 larvae into $20 \%$ sucrose for $3 \mathrm{~h}$ (starved).

\subsubsection{Dissection and Staining of Fat Bodies with LysoTracker Red}

1. Wash larvae thoroughly in water or PBS.

2. Transfer three to five larvae into a drop of PBS. Cut them in half using a sharp blade, and turn the carcasses inside out using forceps.

3. Separate fat body lobes and transfer them to staining solution $(1 \times$ LysoTracker Red + DAPI, prepared fresh daily) for $2 \mathrm{~min}$.

4. Transfer fat body pieces into a drop of PBS or 50\% glycerol-PBS on a slide. Cover with a cover slip and evaluate immediately using a fluorescent microscope.

\subsubsection{Fixation for Confocal Microscopy}

1. Follow the above steps, but dissect in PBS and fix in freshly prepared 3.7\% formaldehyde in PBS for 20 min to overnight at $4^{\circ} \mathrm{C}$.

2. Wash two times in PBS.

3. Mount in Vectashield mounting medium.

4. Seal preparations by applying nail polish around the edges of cover slips and store at $4^{\circ} \mathrm{C}$.

\subsubsection{Fixation and Embedding for TEM}

1. Follow the steps described earlier, but dissect in PBS and fix overnight at $4^{\circ} \mathrm{C}$ in $2 \%$ paraformaldehyde, $4 \%$ glutaraldehyde, $2 \%$ sucrose, $100 \mathrm{mM}$ phosphate buffer.

2. Rinse in $3 \%$ sucrose in $100 \mathrm{~m} M$ PBS.

3. Postfix in $2 \% \mathrm{OsO}_{4}$ in $100 \mathrm{~m} M$ phosphate buffer overnight.

4. Dehydrate in ethanol and embed into Polybed 812 following the manufacturer's recommendations. 


\section{Notes}

1. All experiments are performed at $25^{\circ} \mathrm{C}$ except as otherwise noted.

2. With sufficient numbers of females, egg-collection times can be shortened to as little as an hour, resulting in a more synchronous larval culture.

3. Turning carcasses inside-out requires skill and practice. An alternative is to simply tear larvae open and dissect out the fat body instead.

4. To speed up the LysoTracker Red staining procedure, it is acceptable to dissect larvae directly in staining solution.

5. It is important that the appropriate amount of liquid be used when mounting fat body lobes. Too little liquid will result in crushing cells when the cover slip is applied, whereas in too much liquid, the fat body will not be flat enough for good photographs.

6. LysoTracker Red bleaches very rapidly, so it is essential to minimize exposure time when using this dye. It can be useful to first find the proper focal plane using the UV/DAPI channel.

7. LysoTracker Red fixes poorly in larval tissues, and in most cases its signal is gone by $5 \mathrm{~min}$ of $3.7 \%$ formaldehyde fixation. If you need to work with a fixed tissue (e.g., more time is needed for imaging, or you have a GFP-Atg8 signal also), reduce fixation time to a few seconds to $2 \mathrm{~min}$. LysoTracker signal will be very weak, but you can use an antibleaching agent that way. Another option is to analyze LysoTracker Red staining and GFP-Atg8 signal in different animals using live and fixed tissue, respectively.

8. For TEM, alternate methods of fixation have also been used successfully. Dissected tissues can be fixed in $3.2 \%$ paraformaldehyde, $0.5 \%$ glutaraldehyde, $1 \%$ sucrose, $40 \mathrm{mM} \mathrm{CaCl}$ in $0.1 \mathrm{~N} \mathrm{pH} 7.4$ sodium-cacodylate overnight, rinsed twice in $0.1 \mathrm{~N}$ sodium-cacodylate, postfixed in $0.5 \% \mathrm{OsO}_{4}$ in $0.1 \mathrm{~N}$ sodiumcacodylate for $1 \mathrm{~h}$ at room temperature, dehydrated in ethanol and embedded in Araldyte (Fluka) following the manufacturer's recommendations.

\section{References}

1. Neufeld, T. P. (2004) Role of autophagy in developmental cell growth and death: insights from Drosophila, in Autophagy (Klionsky, D. J., ed.), Landes Bioscience, Georgetown, pp. 224-232.

2. Velentzas, A. D., Nezis, I. P., Stravopodis, D. J., Papassideri, I. S., and Margaritis, L. H. (2007) Mechanisms of programmed cell death during oogenesis in Drosophila virilis. Cell Tissue Res. 327, 399-414.

3. Akdemir, F., Farkas, R., Chen, P., et al. (2006) Autophagy occurs upstream or parallel to the apoptosome during histolytic cell death. Development 133, 1457-1465.

4. Juhasz, G., and Sass, M. (2005) Hid can induce, but is not required for autophagy in polyploid larval Drosophila tissues. Eur. J Cell Biol. 84, 491-502.

5. Martin, D. N., and Baehrecke, E. H. (2004) Caspases function in autophagic programmed cell death in Drosophila. Development 131, 275-284. 
6. Gorski, S. M., Chittaranjan, S., Pleasance, E. D., et al. (2003) A SAGE approach to discovery of genes involved in autophagic cell death. Curr. Biol.13, 358-363.

7. Lee, C. Y., and Baehrecke, E. H. (2001) Steroid regulation of autophagic programmed cell death during development. Development 128, 1443-1455.

8. Lee, C. Y., Clough, E. A., Yellon, P., Teslovich, T. M., Stephan, D. A., and Baehrecke, E. H. (2003) Genome-wide analyses of steroid- and radiation-triggered programmed cell death in Drosophila. Curr. Biol. 13, 350-357.

9. Scott, R. C., Juhasz, G., and Neufeld, T. P. (2007) Direct induction of autophagy by Atg1 inhibits cell growth and induces apoptotic cell death. Curr. Biol .17, 1-11.

10. Scott, R. C., Schuldiner, O., and Neufeld, T. P. (2004) Role and regulation of starvation-induced autophagy in the Drosophila fat body. Dev. Cell 7, 167-178.

11. Lindmo, K., Simonsen, A., Brech, A., Finley, K., Rusten, T. E., and Stenmark, H. (2006) A dual function for Deep orange in programmed autophagy in the Drosophila melanogaster fat body. Exp. Cell Res. 312, 2018-2027.

12. Rusten, T. E., Lindmo, K., Juhasz, G., et al. (2004) Programmed autophagy in the Drosophila fat body is induced by ecdysone through regulation of the PI3K pathway. Dev. Cell 7, 179-192.

13. Ravikumar, B., Vacher, C., Berger, Z., et al. (2004) Inhibition of mTOR induces autophagy and reduces toxicity of polyglutamine expansions in fly and mouse models of Huntington disease. Nat. Genet. 36, 585-595.

14. Scott, P. H., Brunn, G. J., Kohn, A. D., Roth, R. A., and Lawrence, J. C., Jr. (1998) Evidence of insulin-stimulated phosphorylation and activation of the mammalian target of rapamycin mediated by a protein kinase B signaling pathway. Proc. Natl. Acad. Sci. USA 95, 7772-7777.

15. Sass, M., and Kovacs, J. (1977) The effect of ecdysone on the fat body cells of the penultimate larvae of Mamestra brassicae. Cell Tissue Res. 180, 403-409.

16. McGuire, S. E., Roman, G., and Davis, R. L. (2004) Gene expression systems in Drosophila: a synthesis of time and space. Trends Genet. 20, 384-391.

17. Theodosiou, N. A., and Xu, T. (1998) Use of FLP/FRT system to study Drosophila development. Methods 14, 355-365.

18. Hennig, K. M., Colombani, J., and Neufeld, T. P. (2006) TOR coordinates bulk and targeted endocytosis in the Drosophila melanogaster fat body to regulate cell growth. J. Cell. Biol. 173, 963-974.

19. Britton, J. S., Lockwood, W. K., Li, L., Cohen, S. M., and Edgar, B. A. (2002) Drosophila's insulin/PI3-kinase pathway coordinates cellular metabolism with nutritional conditions. Dev. Cell 2, 239-429. 
